Please do not remove this page

RMIT

UNIVERSITY

\title{
Schrödinger's microbe: implications of coercing a living organism into a coherent quantum mechanical state
}

Bull, Joseph; Gordon, Ascelin

https://researchrepository.rmit.edu.au/esploro/outputs/9921862806601341/filesAndLinks?institution=61RMIT_INST\&index=null

Bull, J., \& Gordon, A. (2015). Schrödinger's microbe: implications of coercing a living organism into a coherent quantum mechanical state. Biology and Philosophy, 30(6), 845-856.

https://doi.org/10.1007/s10539-015-9500-4

Document Version: Accepted Manuscript

Published Version: https://doi.org/10.1007/s10539-015-9500-4

Repository homepage: https://researchrepository.rmit.edu.au

(c) Springer Science+Business Media Dordrecht 2015

Downloaded On 2023/04/26 21:05:31 +1000

Please do not remove this page 
Thank you for downloading this document from the RMIT Research Repository.

The RMIT Research Repository is an open access database showcasing the research outputs of RMIT University researchers.

RMIT Research Repository: http://researchbank.rmit.edu.au/

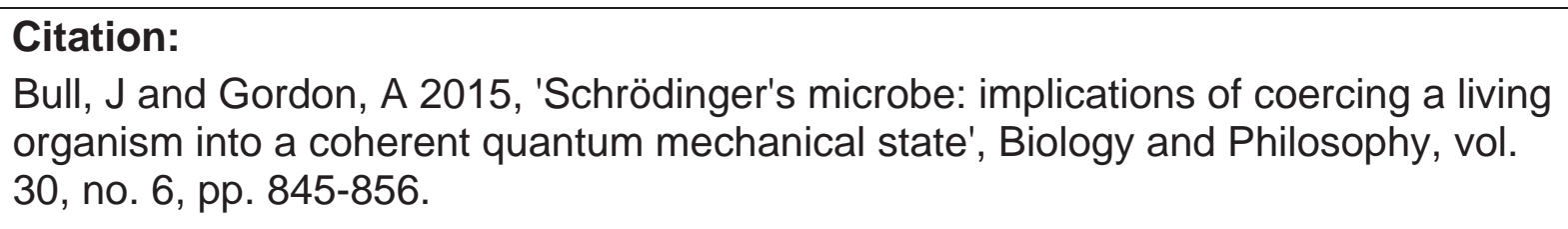
organism into a coherent quantum mechanical state', Biology and Philosophy, vol. 30, no. 6, pp. 845-856.

See this record in the RMIT Research Repository at:

https://researchbank.rmit.edu.au/view/rmit:33935

Version: Accepted Manuscript

Copyright Statement:

(C) Springer Science+Business Media Dordrecht 2015

Link to Published Version:

https://dx.doi.org/10.1007/s10539-015-9500-4 


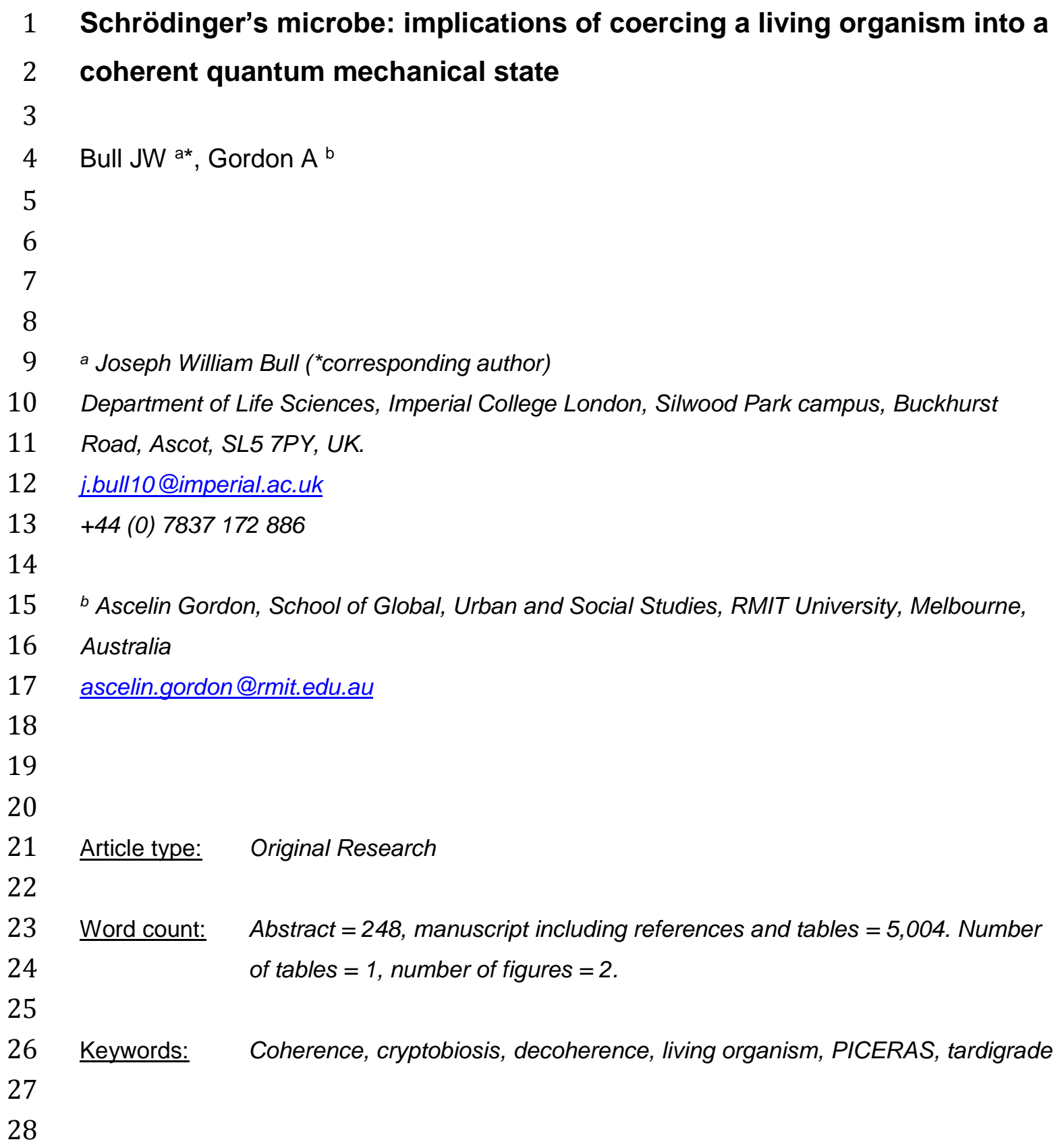




\section{Abstract}

30 Consideration of the experimental activities carried out in one discipline, through the lens of

31 another, can lead to novel insights. Here, we comment from a biological perspective upon

32 experiments in quantum mechanics proposed by physicists that are likely to feasible in the

33 near future. In these experiments, an entire living organism would be knowingly placed into a

34 coherent quantum state for the first time, i.e. would be coerced into demonstrating quantum

35 phenomena.

36

37 The implications of the proposed experiment for a biologist depend to an extent upon the

38 outcomes. If successful (i.e. quantum coherence is achieved and the organism survives after

39 returning to a normal state), then the organism will have been temporarily in a state where it

40 has an unmeasurable metabolism - not because a metabolic rate is undetectable, but

41 because any attempt to measure it would automatically bring the organism out of the state.

42 We argue that this would in essence represent a new category of cryptobiosis. Further, the

43 organism would not necessarily retain all of the characteristics commonly attributed to living

44 systems, unlike the currently known categories of cryptobiosis.

45

46 If organisms can survive having previously been in a coherent state, then we must accept that

47 living systems do not necessarily need to remain in a decoherent state at all times. This would

48 be something new to biologists, even if it might seem trivial to physicists. It would have

49 implications concerning the physical extremes organisms can tolerate, the search for

50 extraterrestrial life, and our philosophical view of animation. 
There is much potential for scientific advancement in interdisciplinary research. However, it is rare for research to be truly interdisciplinary; and so as researchers, we should be watchful for developments in other areas of science that may influence our own. In this article, we discuss what is likely to be just such a development: the implications for biology of specific experiments proposed by physicists. In essence, the proposals are to coerce a living organism (such as a tardigrade - a water dwelling extremophile) into behaving as a coherent quantum object (e.g. Romero-Isart et al., 2010). Whilst there is no apparent theoretical reason that such experiments would not work from a physical perspective - rather, it is a matter of finessing the relevant experimental technology - the implications of the experimental outcomes from a biologist's point of view have yet to be fully considered. Here, after outlining some relevant physics and biology, we discuss the implications of such an experiment for the study of living systems.

\section{Quantum theory and the concept of decoherence}

A key conceptual and philosophical challenge, during the development of quantum mechanics, has been that it is full of strange phenomena that do not intuitively describe the reality we perceive directly around us at a macroscopic scale. Instead, the world we perceive at the macroscopic scale appears to behave more closely in accordance with classical Newtonian mechanics. This challenge can be resolved via the interpretation that macroscopic systems are in what physicists call a 'decoherent' state, as opposed to a state that is 'coherent' i.e. one which clearly exhibits quantum phenomena (Zurek, 1991; 2003). To expand: quantum mechanical phenomena demonstrably hold in laboratory conditions on very small scales for particle systems that are isolated from their environment, and are consequently described by Schrödinger's wave equation. Such particle systems can evolve into a coherent state that is characterized by a wave function, and cannot be considered to actually exist in any one physical state (e.g. being localized to a specific position in space). Rather, all that can be said is that, if measured, the particle system would be found to be in one of various physical states, with probabilities of being found in each state determined by the particle systems 'wave function'. Before measurement, the system can thus be thought of as being in a superposition of multiple possible states at the same time, although it is hard to visualize what this might actually look like. If a measurement is taken of such a particle system, then the probability of the system being recorded in any one of these physical states is related to the squared amplitude of the wave function for that state. The act of measurement, which necessarily involves the particle system interacting with some other system (e.g. the experimental apparatus required to take the measurement), causes the wave function to 'collapse' into one of these single, decoherent, physical states.

\section{As a hypothetical example, imagine a tardigrade that was at an unknown location: if the} tardigrade was in a decoherent state, then an observer could locate it by attempting to measure its position. Subsequently, the observer could legitimately describe the tardigrade as 
having had a defined position in space immediately prior to measurement. But if it were in a coherent quantum state, this would mean it was in a "superposition of states", or, spread out over numerous locations at the same time, with a probability of being found at each. The act of observing the coherent tardigrade (i.e. interacting with it) would have caused its wave function to collapse, with the result that it would decohere and subsequently become localized to a specific point in space (Fig. 1).

In systems we perceive as exhibiting classical behavior, such as most macroscopic systems, the majority of the quantum information about the system is already lost as a result of interactions with the environment ("measurement" being just one form of interaction with the environment). That is to say, the wave function describing such systems is constantly being collapsed into a single decoherent state as a result of these interactions (Zurek, 1991). A decoherent system is indistinguishable from a system behaving deterministically, as described by classical mechanics, which is why macroscopic systems built from components small enough to experience quantum effects don't exhibit this behaviour. For biologists interested in a full introduction to basic quantum mechanics, Davies \& Betts (2002) is recommended.

In order to place an object into a coherent state in the laboratory, it is necessary to isolate it from interactions with its environment. Simplistically, this requires placing the object in a vacuum and cooling sufficiently so that its own internal thermal vibrations do not cause it to decohere. However, it should be noted that the role of interactions disrupting quantum effects is complex, and the fact there is some evidence that living organisms do internally make use of quantum phenomena would imply that quantum effects can occur within warm and nonisolated environments (Ball, 2011; Bordonaro \& Ogryzko, 2013). For the present at least, a practical challenge to coercing objects into a coherent state is that they must be contained within a vacuum and sufficiently cooled - the former to prevent decoherence resulting from interactions with the external environment, the latter to prevent decoherence through thermal vibrational excitation of the object (or of components internal to the object). Such factors limit the size of object that can currently be placed in a quantum coherent state: the larger the object, the more difficult it is to cool and isolate the object sufficiently. A key quantum phenomenon - wave-particle duality - has long been demonstrable in buckminsterfullerenes (C-60), which have a diameter $1 \mathrm{~nm}$ and are 'almost classical' in size (Arndt et al., 1999). As technology continues to improve, it has been possible for physicists to demonstrate coherence in larger and larger objects. More recently, it has been shown that macroscopic inanimate objects, on the scale of $\mu m$, can also be coerced into exhibiting coherent quantum behavior, specifically a superposition of motion states (O'Connell et al., 2010). 
131 Romero-lsart et al. (2010) have proposed an experiment by which lasers would be used to

132 cool (i.e. limit rotational and/or translational motion) and trap a virus, inside what is known as

133 an optical cavity. The virus would be decoupled from its environment and thereby able to be

134 coerced into a coherent quantum state. More specifically, the centre of mass of the virus

135 would be in a superposition of motion states, meaning that the virus was effectively moving

136 (within the confines of the trap) in a number of different ways at the same time. Romero-Isart

137 et al. claim that this "opens up the possibility of testing the quantum nature of living organisms"

138 (i.e. motion as whole quantum objects) such as the common Influenza and Tobacco Mosaic

139 viruses, and potentially larger organisms such as tardigrades. It should be noted that,

140 although the point is not acknowledged by Romero-Isart et al. (2010), there is no consensus

141 amongst biologists as to whether viruses actually comprise living systems (Nasir et al., 2012).

142 However, since the application of the experimental technique is also discussed in relation to

143 tardigrades and other extremophiles, which certainly seem to meet the criteria of being "alive",

144 we do not discuss the virus debate any further.

146 The proposed experiment would result in a living object that is in a superposition of states in

147 relation to e.g. the motion of its centre of mass along one axis. An organism in such an

148 experimental setup would then be subjected to a quantum state, where it would be in a

149 number of different states of motion at the same time, constituting a classically impossible

150 combination of movements. So for instance, unlike a decoherent virus with a certain

151 translational motion and a specific location at a given point in time (Fig. 2A), the coherent

152 virus might be undergoing a combination of translational motions, and thereby also be in an

153 undetermined location in space (Fig. 2B,C).

155 Whether a tardigrade as an organism can be said to "experience" its own movement at all is

156 another topic of discussion, and we do not explore that here. Further, the experimental

157 technique proposed by Romero-Isart et al. has yet to be achieved in practice for objects large

158 enough to comprise a living system, although progress continues to be made towards doing

159 so for inanimate nanospheres (e.g. Kiesel et al., 2013 - who report trapping of submicron

160 particles with a radius of $\sim 169 \mathrm{~nm}$ ), and once it is successfully achieved for larger

161 nanospheres the experiment with viruses is likely to be carried out (O. Romero-Isart, pers.

162 comm.). Nevertheless, the fundamental question that it should inspire for biologists remains

163 worthy of consideration: can living organisms exhibit quantum mechanical properties as whole

164 systems whilst remaining alive, or at least retain the potential to become alive again, and if so,

165 what are the implications? To begin to answer this question, we must first consider some

166 relevant biology - not least the current understanding of a 'living organism'. 
169 A universal definition for what comprises 'living' has yet to be agreed (McKay, 2004), but a common working definition is that an organism is a "self-sustaining chemical system capable

171 of Darwinian evolution" (Benner, 2010). Arguments have been made against this definition

172 (e.g. Ruiz-Mirazo et al., 2004; Leitner \& Firneis, 2011) and others have made attempts to

173 describe life in terms of more specific characteristics. A widely cited set of fundamental living

174 characteristics can be summarized by the acronym PICERAS (Koshland, 2002; Table 1):

175 Program, Improvisation, Compartmentalization, Energy, Regeneration, Adaptability, and

176 Seclusion. Whilst this has been recognized by many (including Koshland) not to represent

177 either a true definition or even necessarily a definitive list of characteristics (e.g. Cleland \&

178 Chyba, 2002), it usefully summarizes a common perception of what a living thing is and does.

179 Note that, because of the requirement to have the capacity to evolve ('improvise' according to

180 Koshland), this set of characteristics applies to whole organisms but not to subcomponents of

181 organisms (e.g. single cells that are not independent). The PICERAS set of characteristics is

182 intended to apply to life at all spatial scales down to the smallest animate objects known to

183 science, which are of the order $300-500 \mathrm{~nm}$. This excludes certain nanobacteria $(\sim 50 \mathrm{~nm})$

184 and viruses ( $10-50 \mathrm{~nm}$ ), which are again not widely accepted to be living organisms (US

185 National Research Council, 1999).

187 The fact that inanimate objects approaching the size of the smallest known living organisms

188 can demonstrably be made coherent - and that certain organisms are known to be able to

189 survive highly extreme conditions, as discussed below - means that it is perhaps inevitable

190 that an experiment such as that proposed by Romero-Isart et al. will soon be carried out. As

191 far as the authors are aware, this would represent an entirely new avenue of study in the field

192 of quantum biology.

\section{Quantum biology}

195 Quantum biology is an emerging discipline, concerned with the extent to which quantum 196 mechanical phenomena are important to, or even purposefully utilized by, living organisms 197 (Ball, 2011). There has for some time been speculation that living organisms internally make 198 use of quantum phenomena (e.g. Penrose, 1989; Hameroff, 1994; Davies, 2004). In order for 199 this to occur, coherence would need to be sustained with the biochemical setting of the living 200 system (Davies, 2004) through a process such as 'internal error correction' (Igamberdiev, 201 2004). Researchers have recently begun to show that this is possible (Gauger et al., 2011), 202 and new research programmes are in progress to examine quantum phenomena at the 203 molecular and cellular levels within biological systems (Bordonaro \& Ogryzko, 2013). Others 204 have proposed the possibility of appropriating mathematical tools from quantum mechanics to 205 model whole ecosystems (Bull, 2015; Rodríguez et al., 2015). However, the Romero-Isart et 206 al. experiment would, for the first time, examine actual quantum effects at the level of a whole organism. It is this latter point that we discuss here, which involves the potential implications 
of coercing a whole living organism (rather than components or sub-components of organisms, such as cells) into exhibiting quantum mechanical behaviour. This topic is important not only to biologists in understanding how living systems function, but also for

211 physicists seeking a better understanding of how to maintain coherence in complex systems

212 (Ball, 2011), and of the so-called 'quantum to classical transition' (Bordonaro \& Ogryzko,

213 2013).

Whilst living organisms are increasingly thought to utilize quantum phenomena, or even to rely upon them by maintaining a level of coherence within subcomponents where necessary, organisms as a whole have only ever been known to behave as classical objects (Davies, 2004; Ball, 2011). That is, whole living organisms have to date never physically been shown to exhibit quantum effects such as e.g. wave-particle duality in a double slit experiment (although this experiment has been carried out on organic molecules; Becker, 2011; Gerlich et al., 2011). By way of explanation: a common version of the double slit experiment finds that,

222 when a coherent electron is fired through a barrier with two adjacent slits, and a detector is later used to monitor which slit the electron passed through, the electron will be recorded by the detector as a discrete 'particle'. However, after many electrons have been fired through the slits, a more general interference pattern will build up on the detector, consistent with a mathematical description of the electron wave functions as having travelled through both slits simultaneously and interfered with themselves (i.e. the electron also acts as a 'wave').

Many physicists, to paraphrase the renowned Anton Zeilinger, would consider the coercion of animate (as opposed to inanimate) objects into a coherent state to be just a question of money and technological innovations - implying it may be of limited interest (Arndt et al.,

232 2005). To biologists, however, there may be more important ramifications of creating living 233 organisms in coherent quantum states. One example, which we discuss here, would be the relevance for the study of cryptobiology.

\section{Cryptobiology}

237 Cryptobiosis (i.e. hidden life) is a state that certain organisms are known to spend time in, and

238 can be defined as "the state of an organism when it shows no visible signs of life and when its metabolic activity becomes hardly measurable, or comes reversibly to a standstill" (Keilin, 1959; Clegg, 2001). A key word in this definition is "reversibly": cryptobiosis requires that the

241 organism can return to a non-cryptic, living state after being, for instance, frozen - rather than

242 expiring. There are five known drivers for a suitably equipped organism to assume a

243 cryptobiotic state: anhydrobiosis (i.e. extreme dessication), anoxybiosis (i.e. in response to a

244 lack of oxygen), chemobiosis (i.e. a response to very high levels of toxins in the environment),

245 cryobiosis (i.e. at very low temperatures), and osmobiosis (i.e. a response to increased levels

246 of solute) (Crowe, 1975). Now, in order to place an organism into a coherent state using a

247 methodology such as that described by Romero-Isart et al., as discussed, it may first have to 
248 be placed in a vacuum and cooled to low temperatures to prevent loss of coherence. The

249 result would be that, in the case of this specific experiment, the organism might assume an

250 anoxybiotic or cryobiotic state (respectively) as a precursor to entering the coherent state.

252 The interesting question from a biological perspective is, then, having potentially already

253 placed the organism into an anoxybiotic or cryobiotic state, does coercing it into a quantum

254 coherent state imply a different category of cryptobiosis? As discussed above, a necessary

255 condition for an organism to remain in a coherent state would be for it to remain isolated from

256 its environment, implying that no measurements could be taken of it. Therefore, it would not

257 be able to have any measurable metabolic activity while in a coherent state (noting that

258 metabolic rate is the rate at which an organism expends energy, which biologists measure in

259 practice through proxies such as rate of gas exchange). The fact that the organism was in a

260 coherent state could be demonstrated without direct interaction or measurement via detection

261 of quantum effects, similarly to the presence of interference patterns found in electrons

262 exposed to the aforementioned double slit experiment.

263

264 Thus, it would have to be concluded that an organism in a coherent state is indeed in a state

265 of cryptobiosis. But this state sets it apart from the other five known classes of cryptobiosis: all

266 of which are states in which metabolic activity can be searched for (e.g. it can be estimated to

267 what degree an organism has managed to expend energy, for instance by assessing how

268 much oxygen it has consumed), but just not physically detected. In a coherent state,

269 metabolic activity cannot be detected - because it is, in principle, impossible to take a

270 measurement without altering the state. A biologist might argue that this conclusion is a

271 question of semantics, but this is because biology tends to treat the act of measurement as

272 something neutral, rather than as an action that physically alters the system being measured

273 (c.f. Fig. 1). Consequently, upon closer inspection, this conclusion may be more profound.

275 Although they do not outline it explicitly, Romero-Isart et al. seem to imply that the experiment 276 could be considered successful if the organism were coerced into being coherent, and then

277 survived the collapse back into a decoherent state. If this is achieved, then the biologist has to

278 conclude that an organism in a coherent state is cryptobiotic - but in a new way compared to

279 previously observed classes of cryptobiosis. This is not the only potentially interesting

280 outcome of the experiment from a biological point of view. In addition, the outcomes have

281 relevance for a PICERAS-type understanding of living things.

283 Compartmentalization

284 The validity of the PICERAS set of characteristics has not, to our knowledge, been fully 285 explored for organisms in a cryptobiotic state. But consider, for instance, an organism that is 286 frozen and hence demonstrates no metabolic activity (i.e. is in a cryobiotic state) - then so 287 long as it may return to an active living state upon warming, it would still exhibit the full set of 
PICERAS set of characteristics (Table 1). It clearly continues to have a Program, is

289 Compartmentalized, and contains Secluded molecules. It cannot demonstrate Improvisation, 290 Regeneration or Adaptability whilst remaining in the cryptobiotic state, but has the capacity to

291 exhibit all three of these characteristics if warmed. Thus a frozen organism has the potential

292 for Improvisation, Regeneration or Adaptability. Similarly, it would require Energy in order to

293 maintain low entropy levels, if it were to return to being a dynamic system or change state in

294 any way, arguably satisfying the last of the 7 PICERAS categories.

296 Almost exactly the same reasoning applies to an organism that is in a coherent quantum state,

297 in the manner proposed by Romero-Isart et al. An organism in a superposition of motion

298 states would similarly still have a Program. Further, it would most certainly have the potential

299 for Improvisation, Regeneration and Adaptability if it could survive returning to a decoherent

300 state. It would retain a latent need for Energy and Seclusion once it lapsed back into

301 decoherence. However, it is possible that whilst the potential for Compartmentalization might

302 be maintained, this characteristic could actually be compromised in such a state. To explain:

303 living systems have a definite boundary, and are also comprised of numerous sub-

304 hierarchical components that themselves have defined boundaries. All known living systems

305 are composed of cells, but these cells might be grouped into organs, and contain organelles.

306 These boundaries are crucial in that they allow matter to traverse them when it is useful to the

307 organism, and also serve to both to keep out undesirable matter and to maintain important

308 chemical processes in isolation (Koshland, 2002). If an entire living system were in a coherent

309 state, it would have no definite internal or external physical boundaries in space. Even if it

310 retained its basic internal structure, in a superposition of motion states, the outer boundary

311 would not be defined in a classical sense. Consequently, normally compartmentalized

312 subcomponents of the organism could in a real sense be considered to be overlapping or

313 non-localised in space, meaning that the characteristic of Compartmentalization had been

314 violated.

316 Again, whilst such an event is perfectly acceptable from the point of view of an inanimate

317 object, it would be a strange state of affairs for a living organism. Whether it is possible for an

318 organism to experience this situation and remain living is, again, one outcome of the

319 experiment that would be worth exploring further. At the very least, a more finessed

320 interpretation of the characteristic of Compartmentalization would be required.

\section{Implications}

323 Here, we have considered certain biological implications of an experimental set up designed

324 by physicists, which would place an organism into a coherent quantum state. The points that

325 arise from a biologist's consideration of the Romero-Isart et al. experiment depend to an

326 extent upon the outcomes. Firstly, if it is successful (i.e. coherence is achieved and the

327 organism remains alive after returning to a decoherent state), then an organism will have 
been temporarily in a state where it has an unmeasurable metabolism: not because a metabolic rate is undetectable, but because any attempt to measure it would automatically bring the organism out of the state. This is in essence a new category of cryptobiosis which to date has been unobserved. Aside from intellectual curiosity, this would be of interest to science and to biologists in particular: because it would extend current understanding of the extreme conditions under which life can persist, and because it would open up a new avenue for exploration in the field of quantum biology.

Secondly, it is not abundantly clear whether the organism could be considered to have demonstrated only partial Compartmentalization, in the sense meant by a biologist, whilst in the coherent state. This would be an interesting avenue for further research, as it would bring into question the validity of characteristics often associated with living things, particularly the assumption that a cellular structure represents a fundamental requirement (Table 1). Whilst it is already accepted by many that we do not have a satisfactory set of characteristics that define an animate organism (Koshland, 2002), such a finding would further shape the debate.

More generally, if it is shown that living organisms can survive being in a coherent state, then we must accept that life does not necessarily require living things to be decoherent - which is in itself a fundamental consideration for biologists, even if it may seem trivial to a physicist. The idea that living things could occupy coherent states would be new to biology, and would perhaps even eventually extend the scope of what is considered possible biologically. By way of just one example that highlights the implications, the field of astrobiology is in part the search for extra-terrestrial life (Morrison, 2001), and a key challenge in that search lies in knowing what exactly to look for (McKay, 2004). Whilst many argue that terrestrial life offers a good template for life elsewhere in the universe (Lineweaver \& Chopra, 2011), it is readily accepted by others that living systems might exhibit entirely different biochemistry to life on earth (McKay, 2011). Given that the definition of life guides the search for it in exotic places, the results of experiments such as the one suggested by Romero-Isart et al. (2010) could influence the exploration for life elsewhere in the solar system.

Finally, and perhaps most intriguing of all, would be if it proved impossible for an organism to resume metabolic activity after being in a coherent state, i.e. if the act of becoming coherent in the proposed experiment always killed it. There is no reason why this should be so from a physical perspective, as far as we know. But it would seem that the two statements:

(1) every object or system in the universe, in principle, can be described by a quantum wave function that is coherent or decoherent to some degree; and,

(2) every living organism that is placed into a coherent state dies, are incompatible. Statement (1) relates to a mainstream interpretation of quantum theory, statement (2) is a potential outcome of the Romero-Isart et al. experiment. If (2) is shown to be true, that would not suggest that quantum theory is misguided - rather, that the current 
368 physical understanding of the universe does not adequately capture animation as a

369 characteristic. That is to say, if it proved to be the case, then it would provide some evidence

370 that living systems have properties that do not fit within our current physical understanding of

371 the universe.

372

\section{Acknowledgements}

374 We acknowledge and thank Leron Borsten and Raimundo Real for in-depth discussions on

375 this topic, and their insights into some of the ideas contained within this manuscript. Jared

376 Cole, Bill Langford and two anonymous reviewers also provided useful comments that

377 improved the manuscript, including suggesting the phrase "Schrödinger's microbe'. J.W.B.

378 acknowledges the support of the Grand Challenges in Ecosystems and the Environment

379 initiative at Imperial College London. A.G. was supported by funding from the Australian

380 Research Council Centre of Excellence for Environmental Decisions. 


\section{References}

Arndt, M., Nairz, O., Vos-Andreae, J., Keller, C., van der Zouw, G. and Zeilinger, A. (1999) Wave-particle duality of $\mathrm{C}_{60}$ molecules. Nature, 401: 680-682.

Arndt, M., Aspelmeyer, M., Bernstein, H.J., Bertlmann, R., Brukner, C., et al. (2005) Quantum Physics from A to Z. arXiv:quant-ph/0505187.

Ball, P. (2011) Physics of life: the dawn of quantum biology. Nature, 474:272-274.

Becker, U. (2011) Molecular physics: Matter-wave interference made clear. Nature, 474:586587.

Benner, S.A. (2010) Defining Life. Astrobiology, 10(10): 1021-1030.

Bennett, C.H. and Shor, P.W. (1998) Quantum Information Theory. IEEE Transactions, 44(6): 2724-2742.

Bordonaro, M. and Ogryzko, V. (2013) Quantum biology at the cellular level - elements of the research programme. BioSystems, 112(1):11-30.

Bull, J.W. (2015) Quantum conservation biology, a new ecological tool. Conservation Letters, DOI: $10.1111 /$ conl.12195.

Clegg, J.S. (2001) Cryptobiosis - a peculiar state of biological organization. Comparative Biochemistry and Physiology Part B, 128: 613-624.

Cleland, C.E. and Chyba, C.F. (2002) Defining 'life'. Origins of Life and the Evolution of the Biosphere, 32: 387-393.

Crowe, J.H. (1975) The physiology of cryptobiosis in tardigrades. Mem Its Ital Idrobiol, 32:3759.

Davies, P.C.W. (2004) Does quantum mechanics play a non-trivial role in life? BioSystems, 78:69-79.

Davies, P.C.W. and Betts, D.S. (2002) Quantum Mechanics: second edition. Nelson Thornes; Cheltenham, UK. 
Gauger, E.M., Rieper, E., Morton, J.J.L., Benjamin, S.C. and Vedral, V. (2011) Sustained Quantum Coherence and Entanglement in the Avian Compass. Physical Review Letters, 106, 040503.

Gerlich, G., Eibenberger, S., Tomandl, M., Nimmrichter, S., Hornberger,K., et al. (2011) Quantum interference of large organic molecules. Nature Communications, DOI: 10.1038/ncomms1263.

Hameroff, S.R. (1994) Quantum coherence in microtubules: a neural basis for emergent

Igamberdiev, A.U. (2004) Quantum computation, non-demolition measurements, and reflective control in living systems. BioSystems, 77(1-3):47-56.

Keilin, D. (1959) The problem of anabiosis or latent life: history and current concept. Proceedings of the Royal Society of London B, 150:149-191.

Kiesel, N., Blaser, F., Delic, U., Grass, D., Kaltenbaek, R., and Aspelmeyer, M. (2013) Cavity

Koshland, D.E. (2002) The seven pillars of life. Science, 295: 2215-2216.

Ladd, T.D., Jelezko, F., Laflamme, R., Nakamura, Y., Monroe, C. and O'Brien, J.L. (2010) Quantum computers. Nature, 464: 45-53.

Morrison, D. (2001) The NASA Astrobiology Program. Astrobiology. 1(1): 3-13.

McKay, C.P. (2004) What is life-and how do we search for it in other worlds? PLoS Biology, 2(9): 1260-1263. and society. Philosophical Transactions of the Royal Society A, 369: 594-606. 
Nasir, A., Kim, K.M. and Caetano-Anolles, G. (2012) Giant viruses coexisted with the cellular ancestors and represent a distinct supergroup along with superkingdoms Archaea, Bacteria and Eukarya. BMC Evolutionary Biology, 12:156.

O'Connell, A.D., Hofheinz, M., Ansmann, M., Bialczak, R.C., Lenander, M., Lucero, E., Neeley, M., Sank, D., Wang, H., Weides, M., Wenner, J., Martinis, J.M. and Cleland, A.N. (2010) Quantum ground state and single-phonon control of a mechanical resonator. Nature, 464: 697-703.

Pati, A.K. (2001) Replication and evolution of quantum species. Fluctuation \& Noise Letters, 4(3): R27-R38.

Penrose, R. (1989) The Emperor's New Mind. Oxford University Press; Oxford, UK.

Rodríguez, R.A., Herrera, A.M., Riera, R., Escudero, C.G., and Delgado, J.D. (2015) Empirical clues about the fulfillment of quantum principles in ecology: Potential meaning and theoretical challenges. Ecological Modelling, 301: 90-97.

Rohde, R.A. and Price, P.B. (2007) Diffusion-controlled metabolism for long-term survival of single isolated microorganisms trapped within ice crystals. Proceedings of the Natural Academy of Sciences of the USA, 104(42): 16592-16597.

Romero-Isart, O., Juan, M.L., Quidant, R. and Cirac, J.I. (2010) Toward quantum

Ruiz-Mirazo, K., Peretó, J. and Moreno, A. (2004) A universal definition of life: Autonomy and superposition of living organisms. New Journal of Physics, 12(3).

US National Research Council (1999). Size limits of very small microorganisms: proceedings of a workshop. National Academies Press; Washington DC, USA. 
501 Zurek, W.H. (1991) Decoherence and the transition from quantum to classical. Physics Today,

502 44(10), 36.

503

504 Zurek, W.H. (2003) Decoherence and the transition from quantum to classical - revisited.

505 arXiv preprint quant-ph/0306072.

506

507 
508 Table 1: Characteristics of living systems, based upon Koshland's PICERAS model of the

509 "pillars of life" (Koshland, 2002)

510

\begin{tabular}{|c|c|c|}
\hline Characteristic & Physical Interpretation & Biological interpretation \\
\hline Program & $\begin{array}{l}\text { Set of instructions } \\
\text { determining behaviour }\end{array}$ & Contained in $R N A / D N A$ \\
\hline Improvisation & $\begin{array}{l}\text { Ability to modify program in } \\
\text { response to environment }\end{array}$ & Evolution \\
\hline Compartmentalization & $\begin{array}{l}\text { Defined boundary, and } \\
\text { isolation of subspaces within } \\
\text { the main system, to } \\
\text { separate processes }\end{array}$ & $\begin{array}{l}\text { Cells as the fundamental unit } \\
\text { of known life }\end{array}$ \\
\hline Energy & $\begin{array}{l}\text { Required for processes and } \\
\text { to maintain low entropy }\end{array}$ & $\begin{array}{l}\text { Living systems consume } \\
\text { energy in low entropy forms }\end{array}$ \\
\hline Regeneration & $\begin{array}{l}\text { Compensate for } \\
\text { thermodynamic losses, } \\
\text { replace missing system } \\
\text { components }\end{array}$ & $\begin{array}{l}\text { Metabolism, replace damaged } \\
\text { biological components }\end{array}$ \\
\hline Adaptability & $\begin{array}{l}\text { Ability to respond to } \\
\text { environment without } \\
\text { changing program }\end{array}$ & $\begin{array}{l}\text { Behavioral change in } \\
\text { response to external stimuli }\end{array}$ \\
\hline Seclusion & $\begin{array}{l}\text { Separation of chemical } \\
\text { pathways }\end{array}$ & $\begin{array}{l}\text { Biological molecules (e.g. } \\
\text { enzymes) are disparately } \\
\text { structured so that they provide } \\
\text { specific functions only }\end{array}$ \\
\hline
\end{tabular}

511

512

513 
514 Figure 1: A schematic illustration of the act of someone observing (A1-3) a normally occurring, 515 decoherent tardigrade, as compared to (B1-3) a tardigrade that is in a coherent superposition 516 of location states. Solid black lines represent the tardigrades wave function. A1: decoherent 517 tardigrade in a specific location. A2: tardigrade is observed (measured). A3: tardigrade is now 518 known to be in that location, but undergoes no physical change. B1: coherent tardigrade is in 519 more than one location simultaneously, with a probability of being observed at each. B2:

520 tardigrade is observed (measured). B3: act of observation causes the wave function to

521 collapse, so that the tardigrade is now decoherent and known to be in one specific location.

522 Tardigrade image modified from Eye of Science/Science Source Images.

524 Figure 2: Schematic illustrating how quantum phenomena might be exhibited if displayed by

525 a virus (grey rectangular shape) in an experiment such as that described by Romero-Isart et

526 al. (2010). (A) decoherent virus in a potential trap, with defined position and known movement

527 along the axis of motion; (B) partially coherent virus in the same trap, movement along this

528 axis is less certain. Possible location is consequently described by a wave function, which is

529 given by the black oscillatory line (the location of the virus staying is the amplitude of the

530 wave function at that point squared); (C) fully coherent virus in the same trap, state of motion

531 along the axis is entirely uncertain until measured. Location is determined proportional to the

532 wave function, which is given by the oscillatory black line. Note that this schematic is

533 conceptually illustrative only i.e. the functional form of the wave function has not been derived. 